\title{
31 \\ Virtual prototyping based development and marketing of future consumer electronics products
}

\author{
P. J. Pulli, M. L. Salmela, J. K. Similä* \\ VTT Electronics, P.O. Box 1100, 90571 Oulu, Finland, \\ tel. +35881 5512111, fax +35881 5512320, e-mail: \\ Petri.Pulli@vtt.fi
}

*CCC Software Professionals and University of Oulu

\begin{abstract}
Industries in many high-volume global businesses, such as automotive, consumer electronics and telecommunications are moving to a two phased product development paradigm with an all-digital front-end to develop the product concepts, and an agile production capability to manufacture and market the product. In this position-type paper we address a particular enabling technology, virtual prototyping, in the context of consumer electronics and telecommunication industries. A virtual reality based development environment for consumer electronics and telecommunication products is described. The aim is to develop all-digital prototypes resembling the physical products as closely as possible in terms of visual 3-D image, behavioural, functional, haptic characteristics and auditory characteristics.
\end{abstract}

Keywords

Consumer electronics, internet, Java, prototyping, virtual reality, VRML

\section{VIRTUAL PROTOTYPING}

A virtual world is a computer generated, often three-dimensional environment in which a user has a sense of presence, where she or he is able to navigate around and can interact with the objects included in it. A virtual 
prototype is defined (Haug et al. 1993) as a computer based simulation of a prototype system or subsystem with a degree of functional realism that is comparable to that of a physical prototype. Virtual prototyping means the process of using virtual prototypes instead of or in combination with physical prototypes, for innovation, test and evaluation of specific characteristics of a candidate design. The following aspects are dominant:

- Functionality: The intended functionality of the prototype that is created virtually is clearly defined and realistically simulated; e.g. product functionality and dynamic behaviour.

- Human Interaction: If human action is involved with the intended functionality of the prototype, then the human functions involved must be realistically simulated, or the human must be included in the simulation; i.e. real-time operator-in-the-loop simulation.

- Environment: If no human action is involved in the intended functionality of the prototype, then either off-line (non-real-time) computer simulation of the functions can be carried out, or a combination of computer (non-real-time) and hardware-in-the-loop (real-time) simulation can be carried out.

In virtual prototyping, a virtual world comprises both the VR model of a product under development, i.e. virtual prototype, and the model of the product's target environment, called virtual target environment. Virtual worlds, the VR models, are highly advanced human-computer interfaces based on Virtual Reality techniques, providing strong intuitive sense of reality and means to change the world through meaningful interaction. Virtual worlds may include various levels of "reality", thus enabling us to concentrate on any desired aspects of the product.

The interaction between the user and the virtual world can be based on different kinds of interaction interfaces (see Figure 1). The most elementary interaction interfaces can be a keyboard and a mouse with a conventional window based 2D user interface. A more sophisticated virtual prototyping environment with 3-D user interface may include e.g. a head mounted display, 3-D position/orientation tracking devices, and auditory as well as haptic feedback devices. The type of representation in a virtual prototyping environment may be spatial, n-dimensional with abstract information spaces, or various combinations of video, natural and computer-generated images.

During the development process, a virtual world can be seen as a model that best matches reality. From the point of view of VR environment, this world can also include the up-to-now implementation, or parts of it. A virtual world thus in fact "replaces" the real world within the development process. 


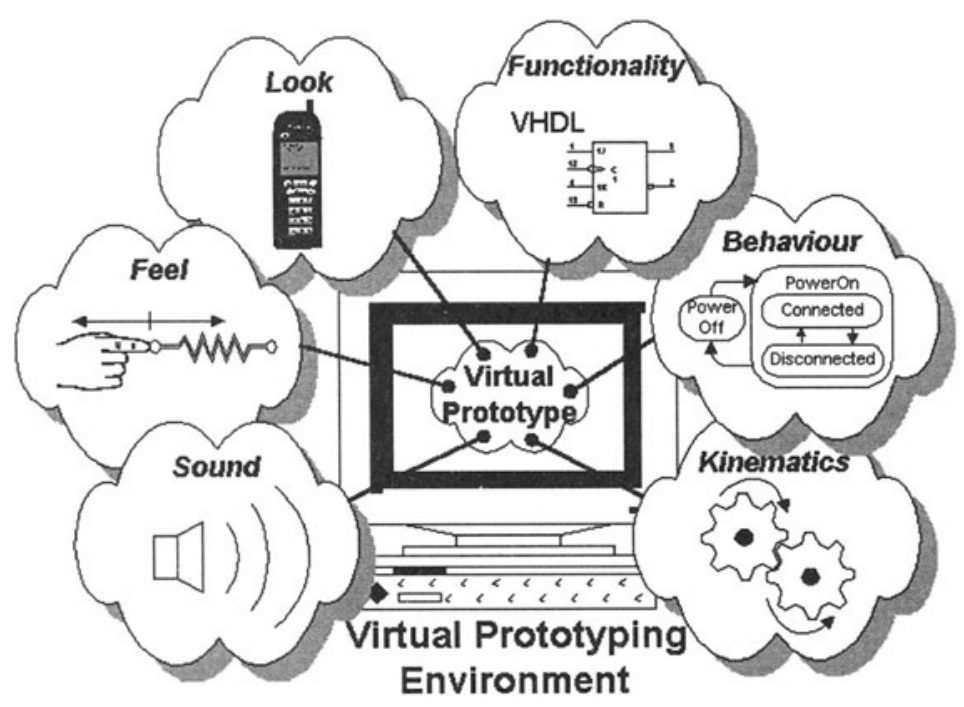

Figure 1 Functionality of typical virtual prototypes for consumer electronics and telecommunication domain.

Virtual prototype worlds might be used, for instance, in:

- Universal combining of different models to improve communication in development projects.

- Prototyping the user observable behaviour and the look/feel aspects of the product that are difficult to specify explicitly.

- Collecting user preferences in "customer clinics" for guiding development towards products with high customer appeal/satisfaction.

- Providing early visibility of a new product in order to demonstrate its capabilities and features.

- Modelling systems operating in hazardous environments, for instance in space, mines or nuclear power plants.

- Modelling systems that cannot be implemented using contemporary technology but which are expected to be feasible in future if technology advances in a predicted rate of progress.

- Providing a virtual salesroom where potential customers may experiment with various product features and ultimately also make product purchases.

A necessary aspect for the full exploitation of the results of virtual prototyping is the requirement for the communication of the results to 
interested parties. These may include persons taking part in actual product development - digital signal processing designers, software and hardware designers, and mechanical designers - or people involved in the sales and marketing of the product - marketing and sales representatives, wholesale and retail representatives, and of course customers. Quite often these persons may be quite widely geographically distributed so solutions based on utilising communication networks are needed.

\section{RESEARCH ENVIRONMENT}

Currently, we are building a research environment to study the previously described virtual prototyping approach applied to consumer electronics and telecommunication products and services. The research environment is illustrated in Figure 2. The environment runs on a Silicon Graphics Indigo2 workstation. A virtual prototype is built by converting the CAD/CAM model specifying the geometry and mechanics of the product into a VR model. Then, the simulation models of the software and hardware parts of the embedded control system are integrated with the VR model to give the virtual prototype the ability to have functionality and behaviour. If necessary, the target environment of the product under development is modelled using VR modelling tools to produce a virtual environment model which is also integrated with the virtual prototype. To achieve an efficient and rapid prototyping capability, a shared database will be used to store reusable VR model components, software components (object-oriented libraries), hardware models, and product data.

The user, either a developer, a customer or a member of any other interest group, interacts with the virtual prototype through various input and output interfaces. In the first phase, we use a conventional 2-D mouse and Logitech 3-D head tracker as input devices. A conventional monitor display with CrystalEyes stereoscopic system (http://www.infolane.com/infolane/ste reog ) is used to provide visual 3-D-feedback. For auditory feedback, stereo headphones and loudspeakers are used. Currently we are in the process of taking into use a haptic interface PHANToM (http://www.tiac.net/users/ sensable) in our environment to provide the user the ability to feel the structure and mechanical functionality of a virtual prototype. At the moment we are limited by the one-finger haptic interface, so we are targeting simple hand-held products. We are also introducing Polhemus tracker (http://www.polhemus.com/trackers/ftrakds.html) for tracking user hands and rapid physical prototypes kept in hand. Figure 2 shows the components of the virtual prototyping environment. Synchronisation of visual, haptic and auditory transactions with the virtual product and its environment is very demanding and requires extended research. 


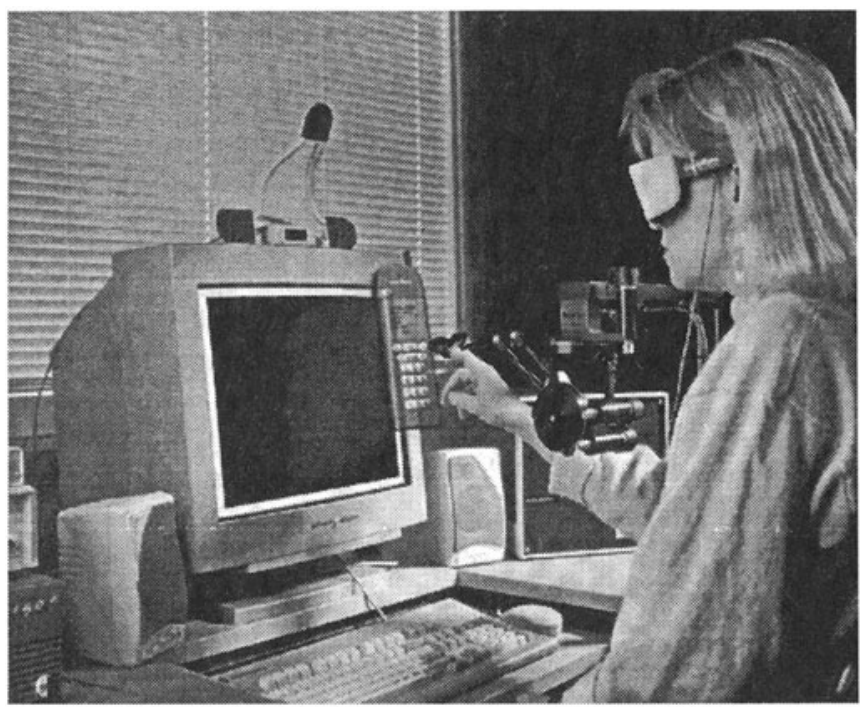

Figure 2 Experimental implementation of part of the virtual prototyping environment for hand-held telecommunication products.

\section{VIRTUAL TELECOM PRODUCTS}

We have chosen to evaluate our approach in three different cases: existing product and its environment, product and service enhancement, and totally new product concept.

In the first case we have studied modelling a virtual cellular phone that has been demonstrated widely to the industry. A limited version of the virtual cellular phone (http://www.ele.vtt.fi/projects/vrp/vrp.html) is also available as a VRML model (http://www.sdsc.edu/vrml/) in Internet. The haptic characteristics need more research to be distributed through Internet. This experiment has given us a much improved position to explain the industry representatives what are the possibilities and limitations of virtual prototyping. We have also found that we need to develop the virtual prototyping environment further in order to improve the realism of the virtual prototype. We are currently integrating our existing software/ hardware simulation tools to the environment. To experiment with heterogeneous prototyping (hardware-in-the-loop), we plan also to connect our virtual prototypes to real software and hardware of a cellular phone. We can also use services provided by the telecommunication network operators. Ultimately, it will be possible to make actual calls using a virtual cellular phone. 
For the second and third cases we have started a research project that tackles the problematics of enhancing an existing product with a totally new kind of accessory. Experience so far indicates that a good place to start introducing virtual prototyping inside industrial companies is to start with industrial designers responsible for product exterior and visual appearance. They seem to be lacking a way to communicate with designer responsible of product interior. We will study the co-existence of virtual and physical prototypes and their relative merits and role in the development process. Because it is possible to develop virtual prototypes of future products and services that cannot be implemented using the technology available today, it is possible to start development projects and market analyses earlier.

\section{VIRTUAL PROTOTYPING TECHNOLOGY IN INTERNET}

Currently our research environment (Figure 2) is based on a stand-alone workstation. This means that in order to utilise the virtual prototyping results in product development we need to have all the persons in the same physical place. In the future in order to account for distributed concurrent product development and to really open up the possibilities for fully utilising the virtual prototyping technology we will experiment with communication solutions based on local area networks and on Internet. In the latter respect VRML (http://www.sdsc.edu/vrml/) combined with Java (http://java.sun.com/) technology seems a viable alternative.

Internet is rapidly expanding from a communication medium to a new market. The present popularity of the WWW as a commercial medium (in contrast to other networks on the Internet) is due to its ability to facilitate global sharing of information and resources, and its potential to provide an efficient channel for advertising, marketing, and even direct distribution of certain goods and information services.

As a commercial medium, the Web offers a number of important benefits which can be examined at both the customer and enterprise levels (Hoffman et al. 1996). Enterprise benefits arise from the potential of the Web as a distribution channel, a medium for marketing communications, and a market in and of itself. Customer benefits arise primarily from the structural characteristics of the medium and include availability of information, provision of search mechanisms, and on-line product trial, all of which can lead to reduced uncertainty in the purchase decision. It is especially the last one, on-line product trial, (cf. e.g. http://www.mathsoft. com or http://www. cox.smu.edu/mis/cases/dec/internet.html) where virtual prototyping would seem a superior technology.

An interesting aspect of the Web is that it seems to offer opportunity for competition on the "speciality" axis instead of the price axis (Hoffman et al. 1996). From a marketing perspective, it is rarely desirable to compete solely on the basis of the price. Instead, marketers attempt to satisfy needs on the basis of benefits sought, which means pricing is dependent upon value to 
the customer, not costs. Such opportunity arises when the offering is differentiated by elements such as convenience through direct electronic distribution of software, or enjoyment through a visually-appealing and exciting Web site. A visually-appealing photorealistic operational virtual 3D prototype of a product would certainly fall in the same category. As evidence that this kind of behaviour is already happening on the Web, consumers indicated that price was the least important product attribute when making on-line purchases (Gupta, 1995). The ability to compete on dimensions other than price will become especially critical in categories where brands are perceived as substitutes (e.g. most hand-held electronic devices), since it allows for more opportunities to differentiate along other dimensions.

Without going into an extensive psychological analysis of the type of persons that would be "thrilled" with the chance of experimenting with virtual prototypes it would seem that the current "leading-edge early adopter" Web users (Booker, 1995) would be likely candidates. They are also a very potential market segment for mobile telecommunication products and services. Already today visual 3-D images of products are accessible through the Internet (http://w3.gti.net/skipjack/). Our approach goes much further than that by providing simulation of close-to-real-life experiences of actually experimenting with and using the products. For the first phase this experimentation would naturally be limited to visual effects and sounds with some simulation of functionality, behaviour and kinematics. The virtual prototypes should be integrated into a commercial Web site comprising an on-line storefront, and a company internet presence (http://www.cox.smu.edu/mis/cases/dec/internet.html).

There are barriers to commercialisation of the Web. Accumulated industry experience, anecdotal evidence as well as personal experience strongly suggest that the main barrier is ease of access. In the context of the Web, ease of access is a multidimensional construct and includes high speed access (the "bandwidth" problem), ease of finding a service provider, and the diffusion of the computer hardware/software/modem bundle into the home (Hoffman et al. 1996). At the moment the high speed access barrier is a serious constraint for using virtual prototypes in the Web since they involve extensive calculations as well as extensive amounts of data to be sent through the network. As mentioned we are investigating a combination of VRML and Java as a possible solution here. The secondary barriers are ease of use, price, and risk, including such factors as privacy and security (Hoffman et al. 1996). At the present, the issue of the security of financial information transmitted over the Internet is impacting customer behaviour on the Web. The majority of consumers use the Web to browse or search much more than to actually purchase something (cf. e.g. Booker, 1995). However, as anyone who follows ordinary newspapers knows, the situation is changing rapidly - for example a recent survey made in Finland indicates that companies expect that electronic commerce will be a general phenomenon in a few years. 


\section{CONCLUSIONS}

We have studied the domain of consumer electronics and telecommunication where we have limited our attention to relatively small products, such as hand-held cellular phones and their accessories. We believe virtual prototyping technology can scale up also to larger products although not necessary using the same kind of virtual reality environments. We expect the Internet technologies (e.g. VRML (http://java.sun.com), Java (http://www.ele.vtt.fi/projects/vrp/vrp.html) to take a big role in future businesses and society. To make possible sales through Internet it is important that the products can be presented in the Internet, i.e. you need to have a fully digital 3-D model of the product and its behaviour and environment. We foresee Internet Virtual Salesrooms. For being able to present the products in virtual salesrooms you have to have a fully digital model of the product. Therefore it is beneficial if the same models that are used in the product design could be used to derive the product models for virtual salesroom marketing.

\section{REFERENCES}

Booker, E. 1995. Web Users Cruising for Info, not Purchases. Computerworld, Feb. 30, p. 29-8.

Gupta, S. 1995. HERMES: A Research Project on the Commercial Uses of the World Wide Web (http://www.mich.edu/ sgupta/hermes/).

Haug, E.J., Kuhl, J.G., Tsai, F.F. 1993. Virtual Prototyping for Mechanical System Concurrent engineering. In Haug E.J. (ed.) Concurrent Engineering: Tools and Technologies for Mechanical System Design. NATO ASI Series Series F: Computer and Systems Sciences. vol. 108. p. 851-879. SpringerVerlag, Berlin Heidelberg.

Hoffman, D., Novak, T., Chatterjee, P. 1996. Commercial Scenarios for the Web: Opportunities and Challenges. To appear in the Journal of ComputerMediated Communication, Special Issue on Electronic Commerce. Also available in http://www2000.ogsm.

\section{BIOGRAPHY}

Dr. Petri Pulli is holding a joint research professor position at VTT Electronics and University of Oulu. He has over 15 years of experience in electronics and telecommunication with special expertise in: concurrent engineering, real-time systems, communication protocols, and virtual reality. Dr. Pulli has over 50 related publications. 